\title{
The Supracerebellar Transtentorial Approach in the Prone Position
}

\author{
Murat KOCAOGLU1 ${ }^{1}$, Feridun ACAR ${ }^{2}$ \\ ${ }^{1}$ Hatay State Hospital, Department of Neurosurgery, Hatay, Turkey \\ ${ }^{2}$ Odak Hospital, Department of Neurosurgery, Denizli, Turkey
}

Corresponding author: Murat KOCAOGLU drkocaoglu@yandex.com

12

To watch the surgical videoclip, please visit http://turkishneurosurgery.org.tr/uploads/jtn-31433-video-1.mp4

\section{ABSTRACT}

The mediobasal temporal region (MTR) is a deep part of the brain covered by eloquent structures. In certain cases, accessing this region is challenging. According to the literature, the supracerebellar transtentorial (SCTT) approach provides safe access to the MTR. Since this approach was introduced by Voigt and Yasargil, many researchers used SCTT in different positions. In particular, the sitting position was the most preferred as it allows the cerebellum to fall away from the tentorium. However, this position has disadvantages such as venous air embolism (VAE), paradoxical air embolism (PAE), and some non ergonomic conditions during surgery. We report two cases with tumors affecting the middle and posterior medial temporal regions. Both patients underwent surgeries in the prone position using the SCTT approach. There were no procedure-related complications. Histopathological results were as follows: psammomatous meningioma in the first case; adenocarcinoma metastasis in the second case. Thus, in this study, the efficacy, feasibility, and safety of accessing the MTR using the SCTT approach in the prone position were demonstrated.

KEYWORDS: Mediobasal temporal region, Supracerebellar transtentorial approach, Prone position

\section{INTRODUCTION}

A ccessing the mediobasal temporal region (MTR) is challenging; this region includes limbic and paralimbic structures. Neocortical structures of temporal and occipital lobes cover the MTR and the fusiform gyrus.

In the literature, various anterior, lateral, and posterior approaches arefoundfor accessing this region $(2,3,14)$. However, reaching MTR is difficult because of the eloquent structures of temporal and occipital lobes. For example, the temporal transcortical approach has a risk of damaging the language function as Wernicke's area is located in the dominant hemisphere. The subtemporal approach has a risk of injuring the vein of Labbé. Moreover, excessive retraction and coursing over these regions are associated with vision defects due to damage of the optic radiation. Furthermore, these approaches are limited due to the restricted operative corridor and combining approaches may be required to see all the MTR (1).

The supracerebellar transtentorial (SCTT) approach is a less invasive and more effective method than the above mentioned approaches. In 1976, Voigt and Yasargil firstly used this approach for removing a cavernous angioma in the posterior hippocampus (17). Then, in 2001, Yonekawa et al. utilized SCTT to clip a distal segment aneurysm of the posterior cerebral artery (PCA) (20). In 2008, Moftakhar et al. used also it for the posterior portion of the MTR (12). In 2012, Ture et al. popularized the technique by using it for selective amygdalohippocampectomy (15).

The SCTT approach can be conducted in sitting, semisitting, park bench, or prone position $(1,4,15)$. However, sitting/ 
semisitting position is generally chosen due to gravity-aided cerebellar retraction $(10,15)$. All these positions have some benefits and/or drawbacks.

Therefore, we will report two illustrative tumor cases using the SCTT approach in the prone position to access the MTR.

\section{ILLUSTRATIVE CASES}

\section{Clinical Presentation}

\section{Case 1}

A 53-year-old man presented with a sudden headache, loss of consciousness, and imbalance. He was admitted with magnetic resonance imaging (MRI) showing a cranial mass associated with the left trigone zone and near the tentorium cerebelli (Figure 1A-C).

\section{Case 2}

A 56-year-old man presented with a history of headache persisting for several weeks with no other positive neurological signs. Brain MRI showed a heterogeneously gadoliniumenhanced lesion on the right MTR (Figure 2A, B).

\section{Surgical Technique}

In both operations, the patients were placed prone using the Mayfield clamp (Figure 3). The approach was planned according to anatomical landmarks and neuronavigation traces (Stealth Stationneuronavigation, Medtronic, Inc., Minneapolis, Minnesota, USA).

A vertical median suboccipital incision was made; after muscles' dissection, posterior fossa craniectomy was performed which extends above the transverse sinus superiorly and above the vermis or $1 \mathrm{~cm}$ more to counter the cerebellar surface and foramen magnum inferiorly. A Y-shaped durotomy was performed. Then, the cisterna magna was opened to release cerebrospinal fluid (CSF), allowing the cerebellum to fall away from the tentorium. After microdissection of the cerebellum from tentorium, an incision on the tentorium was made after confirming the lesion's location using neuronavigation. Then, the tumor was debulked through the tentorium using curettes and an aspirator (Video 1).

After completion of tumor resection under microscopy, we did not suture the tentorium, after hemostasis, as it was opened with a small incision. Water tight dural closure with sutures

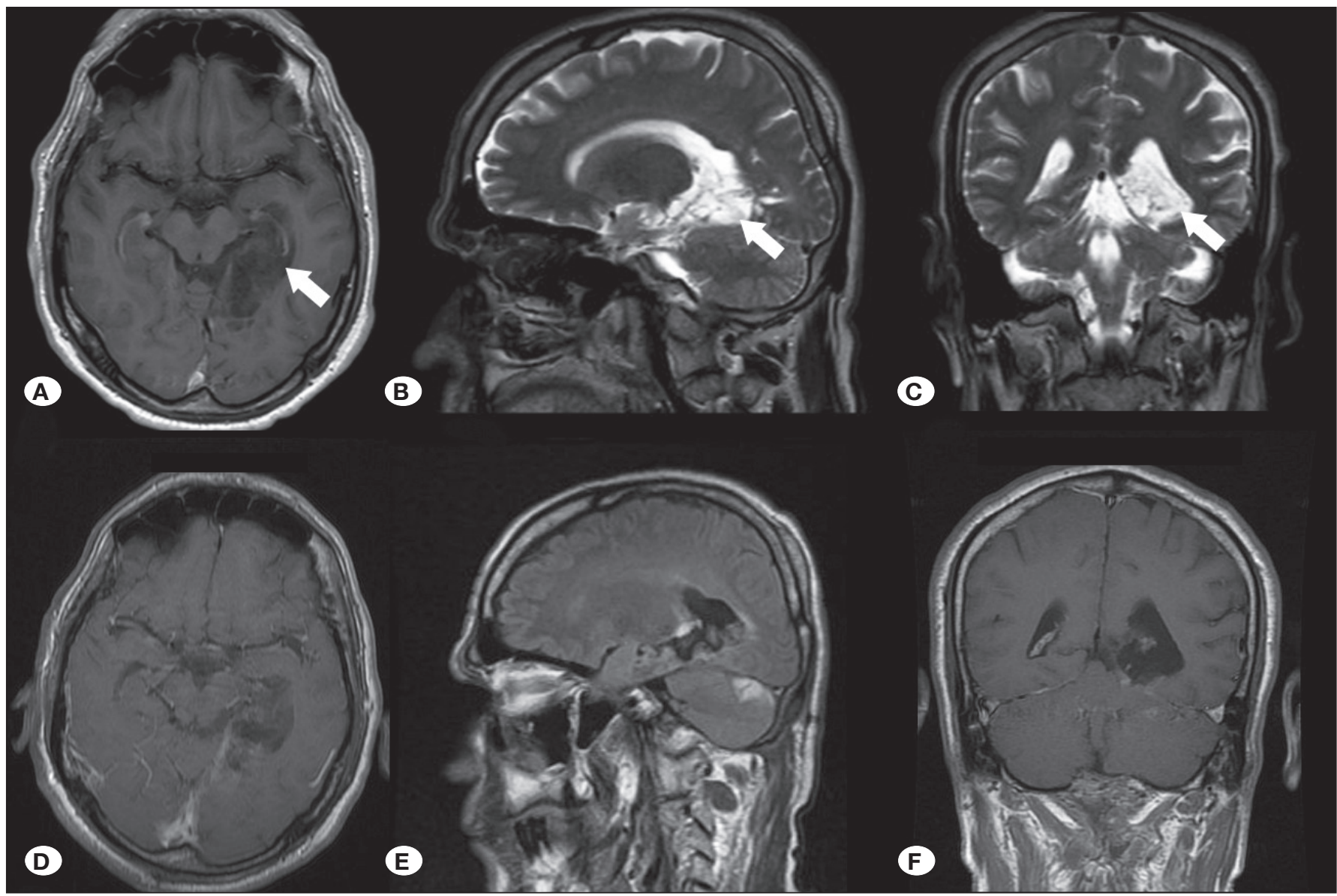

Figure 1: Case 1; preoperative (upper) and early postoperative (lower) images were obtained. A) T1W+C axial MRI shows lesion (white arrow); B) T2W MRI shows lesion and trigone (white arrow); C) T2W MRI shows lesion and tentorium cerebelli (white arrow); D) T1W+C axial MRI; E) T1W+C sagittal MRI; F) T1W+C coronal MRI. 


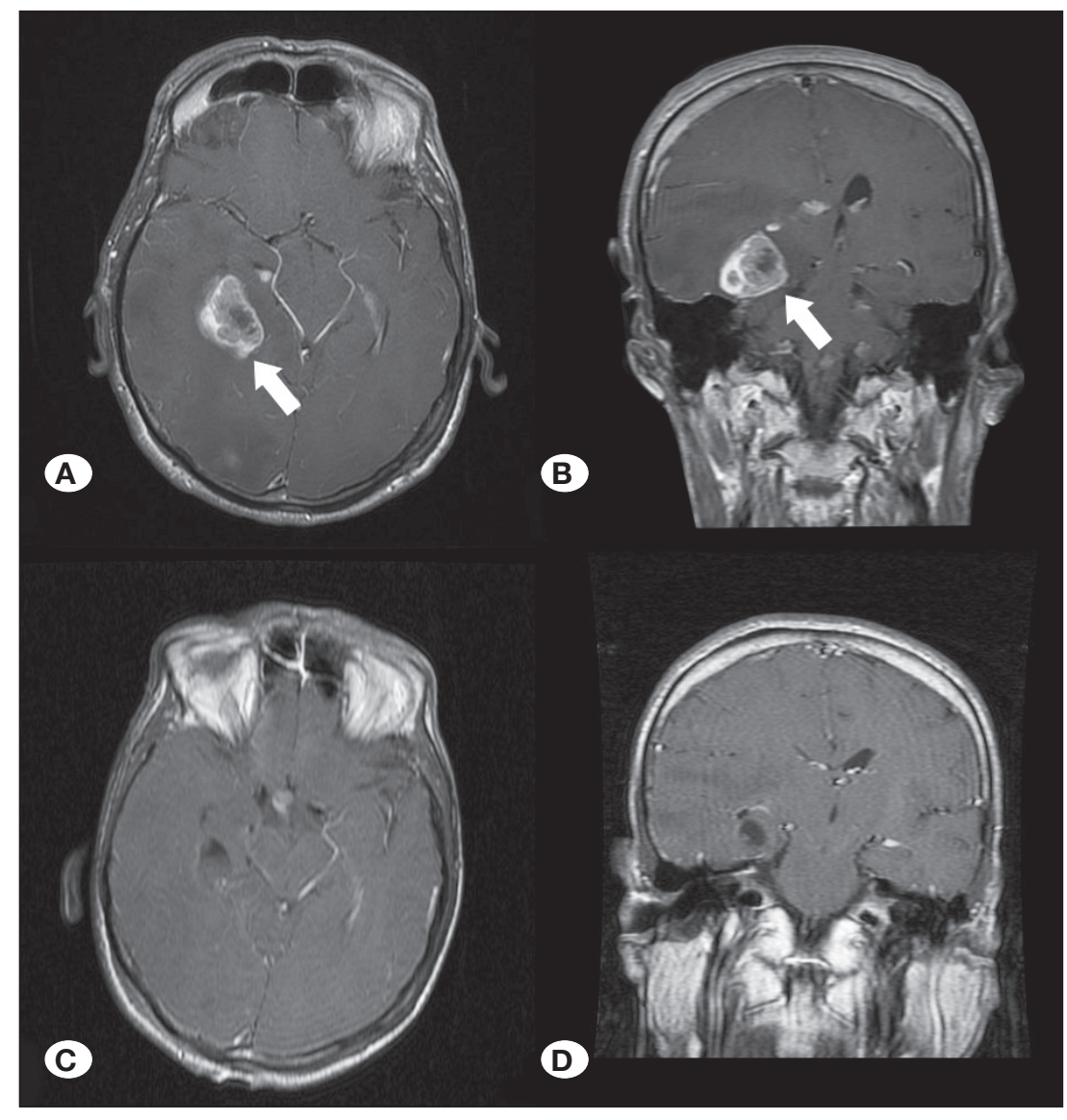

Figure 2: Case 2; preoperative (upper) and early postoperative (lower) images were obtained.

A) $\mathrm{T} 1 \mathrm{~W}+\mathrm{C}$ axial MRI shows lesion (white arrow); B) $\mathrm{T} 1 \mathrm{~W}+\mathrm{C}$ coronal MRI shows lesion (white arrow); C) $\mathrm{T} 1 \mathrm{~W}+\mathrm{C}$ axial MRI; D) $\mathrm{T} 1 \mathrm{~W}+\mathrm{C}$ coronal MRI.

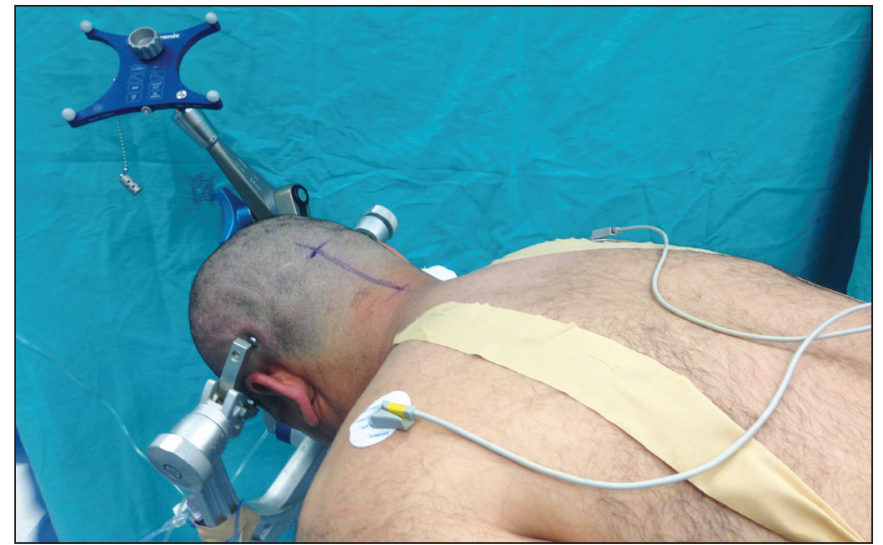

Figure 3: Patient in the prone position.

and fascia was performed. Wound drainage was not used and all tissues were closed layer by layer.

\section{Postoperative Course}

Histopathological investigation revealed psammomatous meningioma in the first one (World Health Organization Grade I) and adenocarcinoma metastasis in the second two.

After surgery, symptoms were normalized and additional neurological deficits were not observed in both patients. Postoperatively, the patients did not suffer from any language or visual deficits. Total resection was achieved and postoperative MRI showed the tumors were excised without brain trauma in both cases (Figures 1A-F; 2A-D).

\section{DISCUSSION}

We report two cases with tumors affecting the middle and posterior medial temporal regions. The studys how the efficacy, feasibility, and safety of accessing the MTR through the SCTT approach in the prone position.

The MTR is the deepest part of the brain surrounded laterally by the collateral sulcus and rhinal sulcus, medially by the carotid, crural, ambient, and quadrigeminal cisterns, anteriorly by the lesser wing of the sphenoid bone, and posteriorly by the isthmus of the cingulate gyrus. The MTR is divided into anterior, middle, and posterior parts (Figure 4). In the anterior part, the uncus, amygdala, hippocampal head, and anterior part of the parahippocampal gyrus are located and the posterior border of the uncus separates the anterior part from the middle part. MTR's middle part includes the parahippocampal gyrus, subiculum, dentate gyrus, fimbria of fornix, and body of the hippocampus and the anterior splenial line separates MTR's middle part from the posterior part. The posterior end of the parahippocampal gyrus is in the posterior part $(9,15,19)$. The SCTT approach was used to access the MTR by many researchers since its introduction by Voigt and Yaşargil (Table I). The sitting and semisitting positions were most preferred, 
Table I: Review of the Literature

\begin{tabular}{|c|c|c|c|c|}
\hline Authors & Year & Lesion & Number of Patients & Surgical Positioning \\
\hline Voigt and Yasargil (17) & 1976 & Cavernoma & 1 & Sitting \\
\hline Uchiyama et al. (16) & 2001 & Tentorial meningioma & 1 & Sitting \\
\hline Moftakhar et al. (12) & 2008 & Ganglioglioma & 1 & Sitting \\
\hline Goel and Shah (7) & 2010 & Epidermoid cyst & 1 & Semisitting \\
\hline Watanabe et al. (18) & 2011 & Petroclival meningioma & 26 & Lateral oblique position \\
\hline de Oliveira et al. (4) & 2012 & $\begin{array}{c}\text { Ganglioglioma } \\
\text { Cavernoma } \\
\text { AVM } \\
\text { PCA aneurysm }\end{array}$ & $\begin{array}{l}3 \\
3 \\
3 \\
3\end{array}$ & Semisitting \\
\hline Marcus et al. (11) & 2013 & Trigone meningioma & 2 & $\begin{array}{c}\text { Sitting } \\
\text { Modified parkbench }\end{array}$ \\
\hline Ansari et al. (1) & 2014 & Tetorial meningioma & 2 & Modified parkbench \\
\hline Weil et al. (19) & 2015 & Glioma & 5 & Lateral parkbench \\
\hline Konovalov et al. (9) & 2015 & Glioma & 20 & Sitting \\
\hline Grigoryan et al. (8) & 2016 & $\begin{array}{c}\text { Glioma } \\
\text { Hemangioblastoma } \\
\text { Cavernoma } \\
\text { Medial temporal sclerosis } \\
\text { Trigone meningioma } \\
\text { AVM }\end{array}$ & $\begin{array}{l}9 \\
1 \\
3 \\
1 \\
2 \\
2\end{array}$ & Sitting \\
\hline
\end{tabular}

PCA: Posterior cerebral artery, SCA: Superior cerebellar artery, AVM: Arteriovenous malformation.

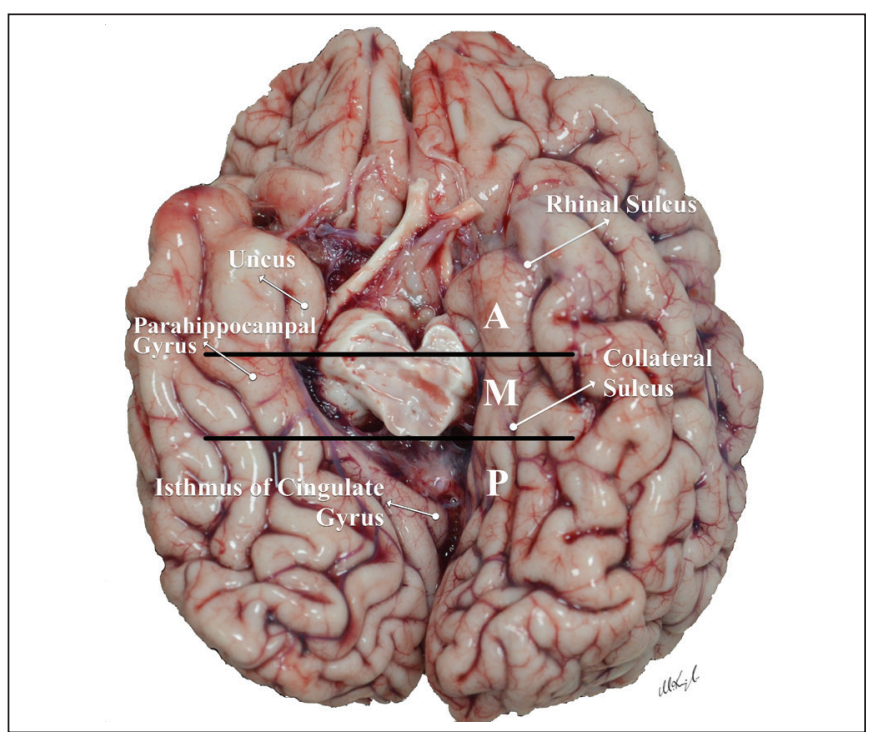

Figure 4: Anatomy of the mediobasal temporal lobe (A: anterior; M: medial; P: posterior). followed by the lateral park bench position, as they allow releasing CSF and the cerebellum to fall away from the tentorium cerebelli. Using the sitting position is still controversial because of the risk of venous air embolism (VAE), and paradoxical air embolism (PAE) (6). VAE incidence during neurosurgery in the sitting position ranges $7 \%-76 \%$ in adults (5). Furthermore, pneumocephalus, macroglossia, quadriplegia, and peripheral nerve injuries were reported in the literature as complications of the sitting position (13).

Moreover, some precautions should be taken during surgery in the sitting position. For example, additional monitoring, including, precordial Doppler, right-heart cath, trans esophageal echo, and esophageal stethoscope, are required to prompt detection and early treatment of VAE. Elastic bandage or antithrombosis socks should be used to prevent venous pooling in the lower extremities against hypovolemia. Furthermore, patients with contraindications for sitting position, such as patent ventriculoatrial shunt and patent foramen ovale, should be evaluated preoperatively. 


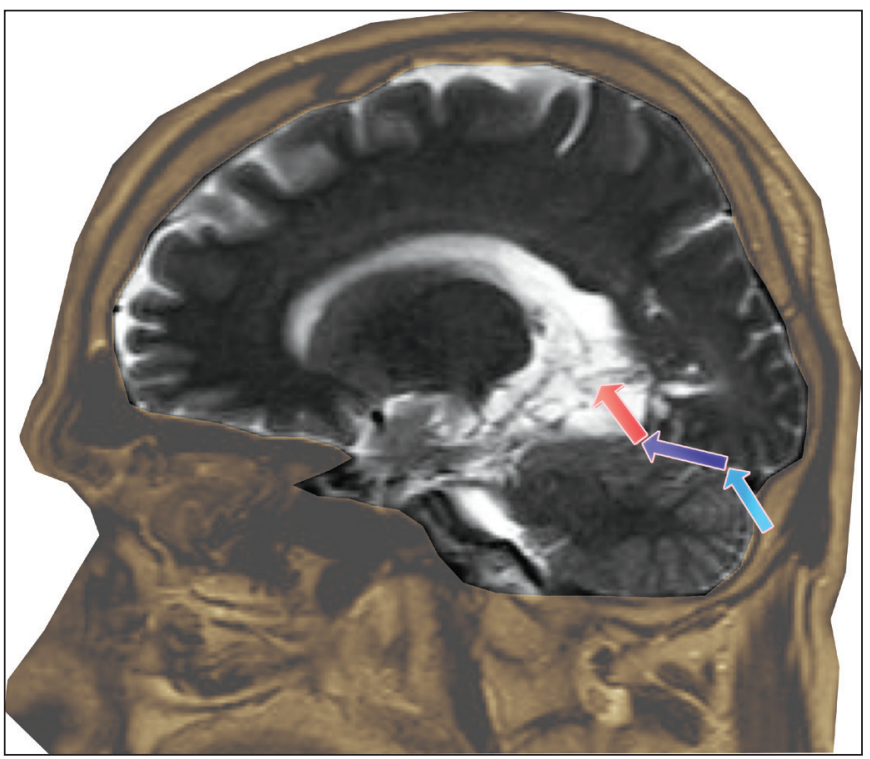

Figure 5: The supracerebellar transtentorial approach for accessing the mediobasal temporal region.

The SCTT approach provides a narrow and deep operative corridor to access. Therefore, the surgeon's comfort and the surgery ergonomics are of great importance in the sitting position, as working above the shoulder level is not comfortable for the surgeon and operating a modern, large optical head surgical microscope can be problematic. Ture et al. have solved the issues by using a surgical microscope with a small optical head controlled by a mouth switch and using an armrest (15).

Previous studies have detailed the SCTT approach $(7,8,11,16,18)$. In this study, a simple and safer variation of this approach that involves placing the patient in the prone position was described. The prone position has a major advantage that is avoiding the risk of potential complications of the sitting position. Moreover, patients with a cardiac shunt (right-to-left) can undergo the operation in the prone position $(15,19)$.

Several points affect the success of the surgery as follows. The tumor's position and the tentorium slope and their relationship with other important structures such as the deep venous system should be carefully evaluated preoperatively. A median incision was made and a standard posterior fossa craniectomy till the foramen magnum conducted. The craniotomy superior limit should be at the inferior edge of the transverse sinus. CSF release from the cisterna magna is important to allow cerebellar relaxation. Increasing density of the venous sinuses in the tentorium midline and posterior parts should be considered while incising the tentorium cerebelli. Therefore, the incision to the midline between the sinus rectus and the lateral edge of the tentorium is made. Hemoclips should be prepared to stop excessive bleeding. Before and after the incision, neuronavigation could determine the shortest route to the lesion. After these steps, reaching the MTR would be easy (Figure 5).

\section{CONCLUSION}

The results of performing the SCTT approach in the prone position in two patients were reported. There wereno procedurerelated complications. Therefore, it can be concluded that the SCTT approach in the prone position is safe, effective, and feasible. This technique will be more applied for larger areas of the brain's deep part with developing technologies such as neuronavigation and maybe robotic surgery in the future. Finally, more cases and long-term follow-up are warranted to endorse the advantages of the prone position for the SCTT approach, especially in the anterior part of the MTR.

\section{REFERENCES}

1. Ansari SF, Young RL, Bohnstedt BN, Gadol AAC: The extended supracerebellar transtentorial approach for resection of medial tentorial meningiomas. Surg Neurol Int 5:35, 2014

2. Bandt SK, Werner N, Dines J, Rashid S, Eisenmen LN, Hogan RE, Leuthardt EC, Dowling J: Trans-middle temporal gyrus selective amygdalohippocampectomy for medically intractable mesial temporal lobe epilepsy in adults: Seizure response rates, complications, and neuropsychological outcomes. Epilepsy Behav 28:17-21, 2013

3. Chang EF, Englot DJ, Vadera S: Minimally invasive surgical approaches for temporal lobe epilepsy. Epilepsy Behav 47: 24-33, 2015

4. de Oliveira JG, Párraga RG, Chaddad-Neto F, Ribas GC, de Oliveira EPL: Supracerebellar transtentorial approachresection of the tentorium instead of an opening-to provide broad exposure of the mediobasal temporal lobe: Anatomical aspects and surgical applications. J Neurosurg 116:764-772, 2012

5. Dilmen OK, Akcil EF, Tureci E, Tunali Y, Bahar M, Tanriverdi T, Aydin S, Yentur E: Neurosurgery in the sitting position: Retrospective analysis of 692 adult and pediatric cases. Turk Neurosurg 21:634-640, 2011

6. Ganslandt O, Merkel A, Schmitt H, Tzabazis A, Buchfelder M, Eyupoğlu I, Muenster T: The sitting position in neurosurgery: Indications, complications and results. A single institution experience of 600 cases. Acta Neurochir (Wien) 155:18871893, 2013

7. Goel A, Shah A: Lateral supracerebellar transtentorial approach to a middle fossa epidermoid tumor. J Clin Neurosci 17:372-373, 2010

8. Grigoryan YUA, Sitnikov AR, Timoshenkov AV, Grigoryan GYU: The paramedian supracerebellar transtentorial approach to the mediobasal temporal region. J Neurosurg 80:43-56, 2016

9. Konovalov AN, Pitskhelauri DI, Melikyan AG, Shishina LV, Serova NK, Pronin IN, Eliseeva NM, Shkatova AM, Samborskiy DYA, Bykanov AE, Golovteev AL, Grinenko OA, Kopachev $\mathrm{DN}$ : Supracerebellar transtentorial approach to tumors of the posterior portions of the medial temporal region. Zh Vopr Neirokhir Im N N Burdenko 79(4):38-47, 2015

10. Manilha R, Harput VM, TureU: The paramedian supracerebellartranstentorial approach for a tentorial incisura meningioma: 3-dimensional operative video. Oper Neurosurg 15(1):102, 2018 
11. Marcus HJ, Sarkar H, Mindermann T, Reisch R: Keyhole supracerebellar transtentorial transcollateral sulcus approach to the lateral ventricle. Neurosurgery 73:295-301, 2013

12. Moftakhar R, Izci Y, Başkaya MK: Microsurgical anatomy of the supracerebellar transtentorial approach to the posterior mediobasal temporal region. Oper Neurosurg 62:1-8, 2008

13. Porter JM, Pidgeon C, Cunningham AJ: The sitting position in neurosurgery: A critical appraisal. Br J Anaesth 82:117-128, 1999

14. Smith KA, Spetzler RF: Supratentorial-infraoccipital approach for posteromedial temporal lobe lesions. J Neurosurg 82:940944, 1995

15. Ture U, Harput MV, Kaya AH, Baimedi P, Firat Z, Ture H, Bingol CA: The paramedian supracerebellar-transtentorial approach to the entire length of the mediobasal temporal region: An anatomical and clinical study. J Neurosurg 116:773-791, 2012

16. Uchiyama N, Hasegawa M, Kita D, Yamashita J: Paramedian supracerebellar transtentorial approach for a medial tentorial meningioma with supratentorial extension: Technical case report. Neurosurgery 49:1470-1474, 2001
17. Voigt K, Yasargil MG: Cerebral cavernous haemangiomas or cavernomas. Incidence, pathology, localization, diagnosis, clinical features and treatment. Review of the literature and report of an unusual case. Neurochirurgia (Stuttg) 19:59-68, 1976

18. Watanabe T, Katayama Y, Fukushima T, Kawamata T: Lateral supracerebellar transtentorial approach for petroclival meningiomas: Operative technique and outcome. J Neurosurg 115:49-54, 2011

19. Weil AG, Middleton AL, Niazi TN, Ragheb J, Bhatia S: The supracerebellar-transtentorial approach to posteromedial temporal Isions in children with refractory epilepsy. J Neurosurg Pediatr 15:45-54, 2015

20. Yonekawa Y, Imhof HG, Taub E, Curcic M, Kaku Y, Roth P, Wieser HG, Groscurth P: Supracerebellar transtentorial approach to posterior temporomedial structures. J Neurosurg 94:339-345, 2001 